\title{
Photon Regeneration from Pseudoscalars at X-ray Laser Facilities
}

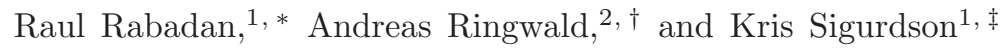 \\ ${ }^{1}$ Institute for Advanced Study, Einstein Drive, Princeton, NJ 08540 \\ ${ }^{2}$ Deutsches Elektronen-Synchrotron DESY, Notkestraße 85, D-22607 Hamburg, Germany
}

\begin{abstract}
Recently, the PVLAS collaboration has reported an anomalously large rotation of the polarization of light in the presence of a magnetic field. As a possible explanation they consider the existence of a light pseudoscalar particle coupled to two photons. In this note, we propose a method of independently testing this result by using a high-energy photon regeneration experiment (the X-ray analogue of "invisible light shining through walls") using the synchrotron X-rays from a free-electron laser (FEL). With such an experiment the region of parameter space implied by PVLAS could be probed in a matter of minutes.
\end{abstract}

Many models beyond the Standard Model predict the existence of new very light pseudoscalar particles which are very weakly coupled to ordinary matter. Such light particles would arise if there was a global continuous symmetry in the theory that is spontaneously broken in the vacuum - a notable example being the axion [1] arising from the breaking of a U(1) Peccei-Quinn symmetry [2], introduced to explain the absence of strong $C P$ violation.

Such pseudoscalars couple to two photons via

$$
\mathcal{L}_{\phi \gamma \gamma}=-\frac{1}{4} g \phi F_{\mu \nu} \tilde{F}^{\mu \nu}=g \phi \vec{E} \cdot \vec{B}
$$

where $g$ is the pseudoscalar-photon coupling, $\phi$ is the field corresponding to the pseudoscalar, and $F_{\mu \nu}\left(\tilde{F}^{\mu \nu}\right)$ is the (dual) electromagnetic field strength tensor. Correspondingly, in the presence of a magnetic field $\vec{B}$, a photon of frequency $\omega$ may oscillate into a pseudoscalar particle of small mass $m_{\phi}<\omega$, and vice versa. The exploitation of this result is the basic idea behind photon regeneration [3, 4] (sometimes called "invisible light shining through walls" experiments). Namely, if a beam of light with $N_{0}$ photons is shone across a magnetic field, a fraction of these photons will turn into pseudoscalars. This pseudoscalar beam can then propagate freely through a wall or another obstruction without being absorbed, and finally another magnetic field located on the other side of the wall can transform some of these pseudoscalars into $N_{f}$ photons - apparently regenerating these photons out of nothing. This type of experiment was carried out in Brookhaven using two prototype magnets for the Collider Accelerator Beam and was used to exclude values of the pseudoscalar-photon coupling $g<6.7 \times 10^{-7} \mathrm{GeV}^{-1}$ for $m_{\phi}<10^{-3} \mathrm{eV}[5]$.

Recently the PVLAS collaboration has reported an anomalous signal in measurements of the rotation of the polarization of photons in a magnetic field [6]. One a priori possible explanation of this apparent vacuum magnetic dichroism is the production of a pseudoscalar coupled to photons through Eq. (10), according to which photons polarized parallel to the magnetic field disappear, leading to a rotation of the polarization plane [7]. The region quoted in Ref. 6] that might explain this signal is $1.7 \times 10^{-6} \mathrm{GeV}^{-1}<g<1.0 \times 10^{-5} \mathrm{GeV}^{-1}$ for $0.7 \times 10^{-3} \mathrm{eV}<m_{\phi}<2.0 \times 10^{-3} \mathrm{eV}$, obtained from a combination of previous limits on $g$ vs. $m_{\phi}$ from a similar, but less sensitive polarization experiment in Brookhaven [5] and the $g$ vs. $m_{\phi}$ curve corresponding to the PVLAS signal. A pseudoscalar-photon coupling in this region of parameter space is in contradiction with limits derived from pseudoscalar production in stars [8], particularly in the sun [9, 10]. However, in principle one can try to find some non-minimal models where pseudoscalar production in stars is small [1] to resolve the discrepancy with the laboratory result.

The main motivation of this note is to suggest an independent laboratory probe of the $g \phi \vec{E} \cdot \vec{B}$ interaction without reference to axion production in stars (see 12, 13]). Given the unexpected and surprising results found in the neutrino sector we believe this type of laboratory crosscheck is certainly warranted. Specifically, we consider the possibility of exploiting a powerful X-ray free-electron laser in a photon regeneration experiment ${ }^{1}$ to probe the region where the PVLAS signal could be explained in terms of a light pseudoscalar particle. ${ }^{2}$

Two facilities have designed and, in fact, are about to commence construction of powerful free-electron lasers (FEL) in the X-ray range: the Linac Coherent Light Source (LCLS) at SLAC 16 and the European X-Ray Laser XFEL at DESY 17]. The LCLS is a free-electron $\mathrm{X}$-ray laser that will use the last kilometer of the SLAC linear accelerator. It will be capable of producing intense pulses of X-ray photons at energies between $0.8 \mathrm{keV}$ and 8 $\mathrm{keV}$. Project completion is expected in 2008 and the first experiments involving the LCLS will be running in 2009. The XFEL at DESY starting in 2012 will have several lasers with similar characteristics with photon energies in the 1-10 keV range and an average flux of photons of approximately $10^{17}-10^{19}$ photons per second. Already running at the DESY TESLA Test Facility is an FEL which

\footnotetext{
1 This idea has been considered first in Ref. 14, with similar sensitivity estimates as in the present note.

${ }^{2}$ For the proposal of another photon regeneration experiment exploiting an ordinary optical laser to test PVLAS, see Ref. [15].
} 


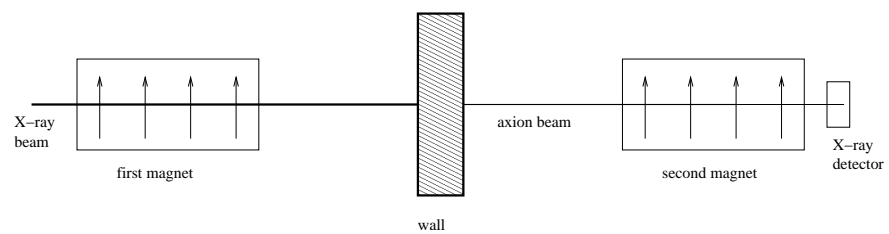

FIG. 1: Schematic figure of the regeneration experiment.

provides tunable radiation from the vacuum-ultraviolet $(10 \mathrm{eV})$ to soft X-rays $(200 \mathrm{eV})$, with an average flux of about $10^{18}-10^{19}$ photons per second [18].

Our benchmark proposal uses a photon regeneration set up with two equal magnets of magnetic field $B$ and length $L$. The first of them converts the X-ray photons from the laser beam into pseudoscalars and the second, on the other side of the "wall", converts the high-energy pseudoscalars into X-ray photons again (see Fig. 1). We consider the sensitivity of two experimental setups: a superconducting magnet of $L=10 \mathrm{~m}$ and $B=10 \mathrm{~T}$ and a conventional magnet with $L=20 \mathrm{~m}$ and $B=1 \mathrm{~T}$. The first setup is more appropriate for the DESY FEL facilities because of the availability of superconducting magnets after the decommissioning of the electron-proton collider HERA in mid of 2007 [19].

The probability of photon-pseudoscalar conversion in a constant magnetic field of length $L$ is:

$$
P=\frac{1}{4} g^{2} B^{2} L^{2} j_{0}^{2}\left(\frac{q L}{2}\right)=g^{2} B^{2} \frac{\sin ^{2}\left(\frac{q L}{2}\right)}{q^{2}},
$$

where $q=\omega-\sqrt{\omega^{2}-m_{\phi}^{2}}$ is the difference between the momentum of the pseudoscalar and the photon. When the mass of the pseudoscalar is much smaller than the photon energy, we can approximate $q=m_{\phi}^{2} / 2 \omega$. For the magnets and pseudoscalar masses we are considering, we have $q L \ll 1$, so that $j_{0} \rightarrow 1$ and the conversion probability simplifies to ${ }^{3}$

$$
P=\frac{1}{4} g^{2} B^{2} L^{2}
$$

Using magnets of a length of $10 \mathrm{~m}=5.07 \times 10^{7} \mathrm{eV}^{-1}$ and a magnetic field of $10 \mathrm{~T}=1.95 \times 10^{3} \mathrm{eV}^{2}$, the probability of converting a photon into a pseudoscalar is

$$
P=2.4 \times 10^{-9}\left(\frac{g}{10^{-6} \mathrm{GeV}^{-1}}\right)^{2}\left(\frac{B}{10 \mathrm{~T}}\right)^{2}\left(\frac{L}{10 \mathrm{~m}}\right)^{2}
$$

\footnotetext{
${ }^{3}$ In Ref. [4] it was claimed incorrectly [20] that the use of a light source of coherence length $\ell_{c}$ degrades the conversion probability by a factor of $\ell_{c} / L$, if $\ell_{c}<L$. This lead to a substantial underestimate of the XFEL sensitivity for photon regeneration in Ref. [19].
}

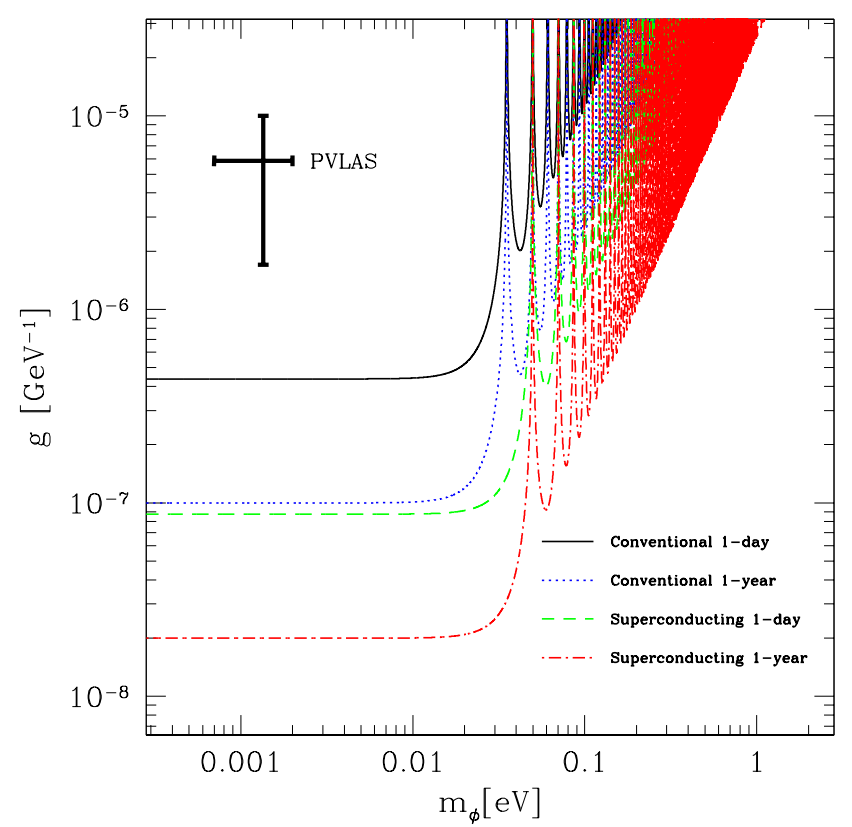

FIG. 2: $95 \%$ confidence level exclusion region for different running times: 1 day and 1 year for two different magnets (a conventional magnet with $L=20 \mathrm{~m}$ and $B=1 \mathrm{~T}$ and a superconducting magnet with $L=10 \mathrm{~m}$ and $B=10 \mathrm{~T}$ ). We have assumed efficient X-ray detection and an X-ray beam with $N_{0}=10^{17} \mathrm{~s}^{-1}$ and $\omega=10 \mathrm{keV}$.

An X-ray laser facility may produce on average as many as $N_{0} \simeq \mathcal{N}_{17} \times 10^{17}$ photons per second (where $\left.\mathcal{N}_{17} \simeq 1-100\right)$. The number of pseudoscalar particles that are produced per second is $N_{\phi}=P N_{0}$. The number that will be transformed back into photons is just $N_{f}=P N_{\phi}=P^{2} N_{0}$. Thus we find the photon regeneration rate as

$$
N_{f}=0.6 \mathrm{~s}^{-1} \mathcal{N}_{17}\left(\frac{g}{10^{-6} \mathrm{GeV}^{-1}}\right)^{4}\left(\frac{B}{10 \mathrm{~T}}\right)^{4}\left(\frac{L}{10 \mathrm{~m}}\right)^{4} .
$$

We immediately see that the PVLAS result can be tested in a matter of minutes. We summarize the full mass dependence of these potential bounds and the values of the pseudoscalar-photon coupling that can be probed in this way for various running times in Fig. 2 For the superconducting magnet and single day experiment, the region $g>8.9 \times 10^{-8} \mathrm{GeV}^{-1}$ could be probed at $95 \%$ confidence, while in a year the limit could be improved to $g>2.0 \times 10^{-8} \mathrm{GeV}^{-1}$.

Let us summarize why we believe our proposal is of interest. Firstly, high frequency photons are able to avoid pseudoscalar-photon incoherent effects, which set in for $m_{\phi}^{2} L \approx 2 \pi \omega$, and can probe pseudoscalar masses ${ }^{4}$

\footnotetext{
${ }^{4}$ Higher masses can be probed by filling in buffer gas into the
} 
$m_{\phi}<0.05 \mathrm{eV}$, beyond the capability of optical photons. Secondly, X-ray detection can be very efficient, with an efficiency $\eta \simeq 1$, and backgrounds in the laboratory are very much under control. They can be reduced by utilizing the directionality of the signal and comparing the backgrounds when the magnets are on with the background when the magnets are off. Thirdly, superconducting magnets are not mandatory and so, in principle, one can use long ordinary magnets that are more cost effective. Moreover, a remarkable feature of this proposal is that, if the initial conversion magnet is placed before a target that is the subject of other experiments, it is possible to perform both experiments simultaneously, since the pseudoscalar beam will propagate unimpeded through the target.

This experiment could probe the region of parameter space relevant to PVLAS in less than a day. Moreover, it could serve as a test facility for an ambitious large scale photon regeneration experiment 19 , based on the XFEL and the recycling of HERA's 400 superconducting dipole magnets $(B=5 \mathrm{~T}, L=200 \times 10 \mathrm{~m})$, to reach within a year an unprecedented sensitivity of $g>2.0 \times$ $10^{-10} \mathrm{GeV}^{-1}$, comparable to the limits involving modeldependent astrophysical considerations [8, 9, 10].

\section{Acknowledgments}

We would like to thank Karl van Bibber, Matthew Kleban, Eduard Masso, Carlos Peña Garay, Pierre Sikivie, Thomas Tschentscher, and Daniel Q. Wang for useful discussions. RR is supported by DOE grant DE-FG0290ER40542. KS is supported by NASA through Hubble Fellowship grant HST-HF-01191.01-A awarded by the Space Telescope Science Institute, which is operated by the Association of Universities for Research in Astronomy, Inc., for NASA, under contract NAS 5-26555.

* Electronic address: rabadan@ias.edu

† Electronic address: andreas.ringwald@desy.de

¥ Hubble Fellow; Electronic address: krs@ias.edu

[1] S. Weinberg, "A New Light Boson?" Phys. Rev. Lett. 40, 223 (1978); F. Wilczek, "Problem of Strong P and T Invariance in the Presence of Instantons," Phys. Rev. Lett. 40, 279 (1978).

[2] R. D. Peccei and H. R. Quinn, "CP Conservation in the Presence of Instantons," Phys. Rev. Lett. 38, 1440 (1977); R. D. Peccei and H. R. Quinn, "Constraints Imposed by CP Conservation in the Presence of Instantons," Phys. Rev. D 16, 1791 (1977).

[3] P. Sikivie, "Experimental Tests of the 'Invisible' Axion," Phys. Rev. Lett. 51, 1415 (1983) [Erratum-ibid. 52, 695 (1984)]; A. A. Anselm, "Arion $<\longrightarrow$ Photon Oscillations in a Steady Magnetic Field. (In Russian)," Yad. Fiz. 42,

magnetic field region [9].
1480 (1985); M. Gasperini, "Axion Production by Electromagnetic Fields," Phys. Rev. Lett. 59, 396 (1987).

[4] K. Van Bibber, N. R. Dagdeviren, S. E. Koonin, A. Kerman and H. N. Nelson, "An Experiment to Produce and Detect Light Pseudoscalars," Phys. Rev. Lett. 59, 759 (1987).

[5] R. Cameron et al., "Search for Nearly Massless, Weakly Coupled Particles by Optical Techniques," Phys. Rev. D 47, 3707 (1993).

[6] E. Zavattini et al. [PVLAS Collaboration], "Experimental Observation of Optical Rotation Generated in Vacuum by a Magnetic Field," arXiv:hep-ex/0507107

[7] L. Maiani, R. Petronzio and E. Zavattini, "Effects of Nearly Massless, Spin Zero Particles on Light Propagation in a Magnetic Field," Phys. Lett. B 175, 359 (1986); G. Raffelt and L. Stodolsky, "Mixing of the Photon with Low Mass Particles," Phys. Rev. D 37, 1237 (1988).

[8] G. G. Raffelt, "Astrophysical Axion Bounds Diminished by Screening Effects," Phys. Rev. D 33, 897 (1986); G. G. Raffelt and D. S. Dearborn, "Bounds on Hadronic Axions from Stellar Evolution," Phys. Rev. D 36, 2211 (1987); G. G. Raffelt, Stars As Laboratories For Fundamental Physics: The Astrophysics of Neutrinos, Axions, and other Weakly Interacting Particles, University of Chicago Press, Chicago, 1996.

[9] S. Andriamonje et al. [CAST Collaboration], "First Results from the CERN Axion Solar Telescope (CAST)," Phys. Rev. Lett. 94, 121301 (2005).

[10] G. G. Raffelt, "Axions: Recent Searches and New Limits," in Proceedings of the Eleventh International Workshop on "Neutrino Telescopes", edited by Milla Baldo Ceolin (Istituto Veneto di Scienze, Lettere ed Arti, Campo Santo Stefano, 2005), pp. 419-431 arXiv:hep-ph/0504152.

[11] E. Masso and J. Redondo, "Evading Astrophysical Constraints on Axion-Like Particles," JCAP 0509, 015 (2005).

[12] A. Dupays, C. Rizzo, M. Roncadelli and G. F. Bignami, "Looking for Light Pseudoscalar Bosons in the Binary Pulsar System J0737-3039," arXiv:astro-ph/0510324

[13] M. Kleban and R. Rabadan, "Collider Bounds on Pseudoscalars Coupling to Gauge Bosons," arXiv:hep-ph/0510183

[14] A. Ringwald, "Fundamental Physics at an X-ray Free Electron Laser," in Proceedings of the Workshop on "Electromagnetic Probes of Fundamental Physics", edited by W. Marciano and S. White (World Scientific, Singapore, 2003), pp. 63-74 arXiv:hep-ph/0112254.

[15] L. Duvillaret, M. Finger Jr., M. Finger, M. Kral, K. A. Meissner, P. Pugnat, D. Romanini, A. Siemko, M. Sulc, J. Zicha, "QED Test and Axion Search by Means of Optical Techniques," CERN-SPSC-2005-034.

[16] Linac Coherent Light Source, http://www-ssrl.slac.stanford.edu/lcls/

[17] European X-Ray Laser Project XFEL, http://xfel.desy.de/

[18] Free-electron laser at TESLA Test Facility, http://www-hasylab.desy.de/facility/fel/main.htm ; "SASE FEL at the TESLA facility, phase 2," DESYTESLA-FEL-2002-01.

[19] A. Ringwald, "Production and Detection of Very Light Bosons in the HERA Tunnel," Phys. Lett. B 569, 51 (2003).

[20] K. van Bibber, Private Communication. 